\title{
Portal Venous Phase Spiral CT
}

National Cancer Institute

\section{Source}

National Cancer Institute. Portal Venous Phase Spiral CT. NCI Thesaurus. Code C19909.

A type of computed tomography (CT) whereby images are taken during the portal venous phase post contrast medium injection. The portal venous phase is defined as the time period in which the contrast medium has passed the heart and the arterial blood vessels and has entered the hepatic portal vein. This type of CT does only apply to liver imaging. 